\title{
El flamenco como herramienta de innovación educativa
}

\author{
Celia Velasco Rodríguez \\ Licenciada en Antropología Social y Cultural
}

\begin{abstract}
El presente artículo es parte del resultado de una investigación doctoral, cuyo objetivo es comprobar la aplicabilidad y efectividad del flamenco como herramienta de intervención socioeducativa. En el texto se muestra el trabajo de investigación realizado sobre la aplicación del flamenco en el ámbito educativo. Se detallan experiencias que han tenido lugar en diferentes espacios educativos formales y no formales de Andalucía. Los resultados obtenidos, arrojan luz sobre las múltiples posibilidades del flamenco como herramienta aplicada a una innovación educativa de éxito.
\end{abstract}

Palabras clave: Flamenco; educación; innovación; cambio; cultura.

This article is part of the result of a doctoral research, the objective of which is to verify the applicability and effectiveness of flamenco as a tool for socio-educational intervention. The text shows the research work carried out on the application of flamenco in the educational field. Experiences that have taken place in different formal and non-formal educational spaces in Andalusia are detailed. The results obtained shed light on the multiple possibilities of flamenco as a tool applied to a successful educational innovation.

1. Antes de comenzar... ¿qué entendemos por flamenco?

Para entender el flamenco actual, es necesario hacer un breve recorrido a lo largo de su historia y del marco teórico elaborado por las investigaciones que han estudiado este fenómeno. Desde su origen, a finales del S XVIII, el flamenco ha pasado por varias etapas: la etapa preflamenca, el café cantante, la ópera flamenca, el neojondismo y el flamenco actual.

Así mismo, hay dos corrientes de pensamiento: una, que incluye a autores como Steingress (2006), Lavaur (1999) o Wasahbaugh (2005), que entiende el flamenco como un fenómeno artístico; otra, representada por Cruces (2002) o Moreno (2012), que lo interpreta como fenómeno cultural.

La etapa preflamenca es el momento previo en el que se sientan las bases y se tienen las primeras noticias sobre este fenómeno musical. El término flamenco engloba un conjunto de elementos estéticos, sociales y culturales que se dan en la sociedad española entre los siglos XVIII-XIX. En este momento tiene lugar un agitanamiento social, es decir una aproximación a la estética y comportamiento de los gitanos. Este movimiento llamado majismo se extiende por diversos géneros artísticos como la literatura, la música o la pintura: «los andaluces pobres abrazaron un conservadurismo resistente que, irónicamente se asemejaba al conservadurismo opresivo y coercitivo del siglo anterior [...] se remodelaron como majos, es decir como seres gallardos y descarados seguros de sí mismos» (Washabaugh, 2005, p. 73).

La estética flamenca cristaliza por una sucesión de hechos históricos como la crisis del antiguo régimen y la creación del estado moderno carente de un proyecto de identidad nacional. Según Lavaur (1976) y Steingress (2006) el flamenco es un género artístico moderno del siglo XIX,

\footnotetext{
*Miembro del Grupo de Investigación EtnoCórdoba Estudios Socioculturales (UCO). Estudiante Plan de Doctorado en Ciencias Económicas, Empresariales y Sociales de la Universidad de Sevilla.
}

Revista de Investigación sobre Flamenco "La madrugá" 
(C) Servicio de Publicaciones de la Universidad de Murcia

http://revistas.um.es/flamenco

que nace en el marco del romanticismo para aportar una identidad cultural al proyecto político nacional, representa los valores románticos que son apreciados por intelectuales modernos, a la vez que abraza la tradición, creando este fenómeno moderno-tradicional y dando pie a su principal ironía (Washabaugh, 2005). En definitiva, el romanticismo impulsa al flamenco como fenómeno artístico, urbano y moderno. No es un arte popular sino popularizado (Lavaur, 1976) y populista (Aix, 2014).

Entre 1850 y 1925 el flamenco se emplea como término para identificar un género musical que se profesionaliza en Cafés Cantantes, configurando la estética flamenca tanto en el cante como en el toque y el baile. Suponen uno de los hitos del flamenco a través del cual, se llevaron a escena las voces de la vida cotidiana andaluza que fueron adaptadas, los cantes refinados y las letras dulcificadas (Washabaugh, 2005). Finalmente tomó más fuerza el cante del café, que el cante de la calle, que fue «ensombrecido por el sonido profesional» (Washabaugh, 2005, p. 76). El café cantante dio lugar a la profesionalización del flamenco como fenómeno artístico y de los artistas que lo interpretaban. Se llegó a crear un mercado artístico especializado a la vez que un foco de vida bohemia que atraía una variedad de actividades, así como la censura de intelectuales y políticos. A partir de 1880, llevaron a cabo una campaña antiflamenca desde una perspectiva modernizadora, por la cual se rechaza lo antiguo y se asocia el flamenco a la delincuencia, nocturnidad y atraso. Sumado a ello, ciertos intelectuales y aficionados rechazan la intensa profesionalización del flamenco de principios del Siglo XX.

Entre los años 1925 y 1955, tiene lugar otra etapa histórica llamada Ópera flamenca. En este período se dan dos tipos de representaciones flamencas: las dirigidas a los aficionados, que se desarrollan habitualmente en los colmaos y son pagadas por señoritos a modo de fiesta privada; y, por otra parte, espectáculos para el gran público en los que se mezclan las formas flamencas con música nacional, como la copla. Como reacción a la decadencia de la ópera flamenca, Manuel de Falla y Federico García Lorca, organizan el Concurso de Cante Jondo en 1922 en Granada, a través del cual pretendían rescatar las tradiciones auténticas que la ópera estaba destruyendo. Sin embargo, el impacto conseguido fue más bien el contrario, pues hay quien piensa que constituyó el «verdadero momento de despegue de la ópera flamenca (Cobo, 1994, p.107)» (Washabaugh, 2005, p. 79).

Desde la perspectiva del flamenco entendido como fenómeno artístico, diferentes autores aluden al hecho de que el flamenco ha sido usado por el estado como "proveedor etnicitario" (Florido y Reigada, 2015, p. 224) para alcanzar sus propias metas. Lavaur (1976) plantea, así, que el flamenco es un fenómeno artístico creado por una estrategia empresarial, en respuesta a temas tradicionales y gitanos demandados por el Romanticismo. Durante el siglo XIX el flamenco aportó a la consolidación del proyecto nacional del estado, al proporcionar una cultura popular que permitiese la unión social (Steingress, 2006). El llamado "nacional-flamenquismo" durante el régimen franquista, mantenía el nacionalismo español anterior a la vez que vendía una imagen simbólica de España de gran aceptación por el turismo incipiente, «y es que la mercantilización ha sido otra posibilidad de instrumentalización, presente en el flamenco desde su propio origen como género artístico» (Florido y Reigada, 2015, pp. 224-225).

Una nueva etapa denominada neojondismo que supuso para el flamenco alcanzar un «estatuto de autonomía artística» (Aix, 2014, p. 45), tiene lugar entre 1955 y 1980 como una reacción purista. En ella se da un movimiento en defensa del flamenco jondo, sus elementos y fundamentos musicales como una reacción a la vulgarización del flamenco en producciones anteriores y usos políticos ligados al franquismo. Se trabaja por integrar de nuevo el flamenco a su raíz gitana, y recuperarlo como modelo estético y cultural debido a la alta producción bibliográfica de varios intelectuales, algunos de los cuales entendían el flamenco como una vía de reacción al franquismo. Tres acontecimientos son claves en esta etapa, por una parte, la publicación de los libros Fla- 
mencología de Anselmo González Climent (1955) y Mundo y formas del cante flamenco (1963) de Ricardo Molina y Antonio Mairena. Otro de los hechos importantes que marcan la etapa es el Concurso Nacional de Cante Jondo de Córdoba, en 1956. Y por último la Antología del cante flamenco publicada por Hixpavox (1954). Musicalmente hablando, en esta etapa se recuperan los cantes básicos, los cantes gitanos, aquellos de mayor pureza y hondura. Hasta los años sesenta los espectáculos tienen lugar principalmente en los Tablaos, como una representación de las fiestas de cabales para turistas, o bien en los Concursos Nacionales de Cante organizados desde 1958 por la Cátedra de Flamencología de Jerez.

Desde 1980 hasta la actualidad comienza una etapa denominada Nuevo Flamenco. En este momento tiene lugar un proceso de mercantilización en el que cobra especial importancia la profusión discográfica, se clasifica al flamenco como música étnica y se da una apertura en mercado discográfico internacional, de manera que llega a tener proyección mundial. A mismo tiempo aumenta el apoyo institucional, político y financiero despertando interés en espacios culturales, audiovisuales o académicos.

En esta época es común la fusión del flamenco con otros géneros como el rock, pop, jazz, etc., se incluyen coros y nuevos instrumentos como el cajón o la flauta. La guitarra cobra protagonismo por sí sola y los palos asociados a la fiesta son los más interpretados. El baile evoluciona creando propuestas complejas de escenografía y aproximando la distancia entre hombres o mujeres.

Es significativa la popularización del flamenco a través de grandes figuras que se convierten en símbolos y fenómenos sociales como Camarón o Paco de Lucía. Aix (2015) relaciona el flamenco con los campos del poder y centra este análisis en tres operaciones: la promoción externa del flamenco, la estructura interna, y el análisis de la génesis de los hábitos de los ocupantes de estas posiciones. Señala también que, en esta última etapa, la institucionalización e instrumentalización del flamenco deriva en la dependencia de los recursos públicos, dando un paso de la revalorización a la sobrevaloración institucional del flamenco, lo cual amenaza la autonomía del flamenco y facilita su decadencia como arte (Aix, 2015, p. 316).

$\mathrm{Al}$ margen de la descripción anterior que entiende el flamenco como fenómeno artístico, otros autores han hecho una lectura etnicista, entendiendo el flamenco como un fenómeno cultural vinculado a prácticas sociales y culturales: fiestas, comunicación, relaciones, etc., al tiempo que a los grupos étnicos presentes en Andalucía durante los siglos XVI y XVII como moriscos, gitanos y clases populares. Este punto de vista es compatible con la teoría del Romanticismo, y permite explicar la singularidad musical del flamenco al vincularlo a formas orientales, así como adelantar los orígenes a una época premoderna, y dar espacio a otras formas preexistentes de música y sociabilidad ligadas a ritos de paso, reuniones y fiestas. En definitiva, explica el proceso histórico de creación de un sistema músico- social por tradiciones culturales propias de grupos sociales y étnicos específicos (moriscos-gitanos-grupos populares).

Desde la perspectiva del flamenco como fenómeno cultural, se definen los rasgos culturales que nos permiten la identificación del flamenco como sistema y patrimonio cultural. En relación al sistema cultural, diferenciamos dos miradas, una etnicista que liga el flamenco a Andalucía y se refiere a rasgos como la sociabilidad, el trabajo o las fiestas, y otra universalista que entiende el flamenco como expresión cultural. Cruces (2002) justifica el arraigo del flamenco en Andalucía, debido a que esta tierra comparte rasgos de sociabilidad con el flamenct 1 .

si bien es verdad que el flamenco refleja muchos de los rasgos que adquieren las relaciones sociales grupales características de la cultura andaluza (la tendencia a la segmentación social,

\footnotetext{
${ }^{1}$ Entendemos sociabilidad como sistema de relaciones sociales institucionalizado: lineamientos, contextos, valores, tipos de expresión, agrupamientos, ritualización, etc.
} 
(C) Servicio de Publicaciones de la Universidad de Murcia

http://revistas.um.es/flamenco

el dominio del componente masculino en los contextos entendidos como públicos), ciertas formas de interrelación propias son privativas de colectivos, espacios y tiempos sociales que se han dado en llamar específicamente flamencos (p. 23).

Mantiene la autora que desde el estudio de la sociabilidad podemos conocer «por qué el flamenco surge y se desarrolla en Andalucía y no en otras sociedades debido a las peculiares formas de relación a través de las cuales se comprende y manifiesta la sociabilidad andaluza» (ibidem). En relación a esta hipótesis, Cruces plantea un análisis metodológico de los rituales flamencos desde el análisis de tres variables (i) nivel de institucionalización y formalización, (ii) participación y vínculos y (iii) la distinción entre el valor de uso y el valor de cambio de la expresión flamenca, (flamenco privado o público) según exista o no un intercambio monetario que responda a la lógica de mercado, o por el contrario responda a una necesidad social como el acompañamiento a una boda o a un trabajo agrícola. Los espacios privados son más dados a la «vivencia completa y socializada del flamenco» (Cruces, 2002, p. 33). Sin embargo, lo significativo es que las relaciones directas no solo se dan en el flamenco privado, sino también «con una dosis de artificio simbólico en muchos casos en peñas o festivales [...] y que constituyen muestra de los momentos sociales formalizados en los cuales el flamenco tiene valor de cambio» (Cruces, 2002: 34). Mantiene esta autora que el flamenco crea y sirve a las relaciones igualitarias, al menos en lo simbólico, ya que en realidad tienden a la segmentación, de igual modo que en la sociedad andaluza, en la que existen multitud de pequeños grupos poco abiertos al exterior, dentro de los cuales se da una sociabilidad generalizada. En este sentido hay que considerar el nivel de acceso al rito flamenco, ya que, si bien el espectáculo es accesible, «el mundo de los flamencos es difícilmente permeable» (Cruces, 2002, p. 35). Para acceder a este mundo es precisa la afinidad, es decir tener la cualidad de ser flamenco, lo cual asegura que se conozcan y respeten los códigos flamencos para la escucha y el disfrute. Otros criterios de segmentación son la etnicidad en cuanto a la categoría de gitano o payo, y el género:

A nadie se oculta que el control patriarcal ha apartado a multitud de mujeres gitanas de la posibilidad de convertirse en profesionales [...] Mientras que la participación de las mujeres gitanas en sus rituales flamencos privados es de pleno derecho, la condición invisible de las mujeres en el flamenco es casi exclusiva de la población no gitana (Cruces, 2002, p. 42).

En el ámbito de las peñas, «resulta difícil encontrar mujeres en las listas de socios o grupos de mujeres que acudan solas a un festival»(Cruces, 2002, p. 43). Los socios de las peñas son hombres en su mayoría, las mujeres simplemente acompañan al marido siendo aficionadas de segunda y meras acompañantes. De modo que estas agrupaciones se configuran desde una mirada masculina y favorecen la participación de los hombres, al planificar los actos en momentos de trabajo familiar o de cuidados en los que las mujeres no pueden asistir. En definitiva, debido a una construcción cultural de los géneros, las mujeres no participan del mismo modo que los hombres ni en peñas, ni en el ámbito artístico, aunque en el baile la presencia femenina ha sido una constante, ha estado marcada por categorías asociadas a la sensualidad y gracilidad. Frente a esto, las mujeres en los hogares han sido las primeras transmisoras del flamenco, que se ha desplazado por matrilinealidad.

Otra de las cuestiones a destacar es la reafirmación de la identidad local en el flamenco: el fuerte peso de la comunidad local es una de las dimensiones para la construcción de fronteras de la identidad en Andalucía. Este componente está muy marcado en el flamenco y condiciona sus modelos de acción ritual, en aspectos tan diversos como la confección de carteles, el predominio de cierto tipo de actividades sobre otras, o las muestras comunicativas entre las partes presentes en las convocatorias (Cruces, 2002, p. 48). 
Lo más interesante en este sentido son los procesos comunicativos que se dan entre artista y público, porque «ahí se exterioriza el vínculo de identificación local y la dimensión comunitaria e integradora» (Cruces, 2002, p. 48) de ciertos eventos flamencos como los festivales. De este modo, «el flamenco permite establecer un proceso de comunicación a través de sus armas estéticas y su plasticidad emocional [...] también de la fiesta, de la identificación comunitaria en la que el arraigo de lo local es indiscutible» (Cruces, 2002, p. 53).

Por tanto, desde la perspectiva del flamenco como fenómeno cultural, los rituales flamencos «van más allá de lo puramente cognitivo o emocional. Sirven de marco para la expresión de la sociabilidad colectiva [...] permiten el establecimiento de redes y relaciones de poder y prestigio y son contextos sociales en que se ponen claramente de manifiesto los vínculos identitarios» (Cruces, 2002, p. 54).

En relación al patrimonio cultural, autores como Isidoro Moreno hacen una revisión crítica de la política llevada a cabo en este ámbito en un sentido de instrumentalización del flamenco a favor de la política o del mercado. El flamenco es marcador de la cultura andaluza, aunque en su análisis se plantean falsos binarismos que obstaculizan esta relación, tales como el gitanismo frente al antigitanismo: andaluces gitanos y andaluces no gitanos. Duda del carácter popular o no popular, frente al que entendemos un flamenco tradicional moderno, compuesto de elementos elitistas y populares. La noción de hermetismo del flamenco, que había sido invisibilizado hasta su mercantilización. Y, por último, una falsa identificación individualista del flamenco que en su esencia es acción colectiva, comunicativa, ritual, experiencia íntima e individual (pero no individualista).

Una cuestión que ha limitado el flamenco ha sido su identificación con lo nacional y español, en esa contribución étnica ya comentada que permitió al sistema político articular el sentimiento nacional, que mencionan autores como Steingress o Aix, desde la mirada del flamenco como fenómeno artístico. Esta cultura nacional inventada, no tiene en cuenta las diferentes realidades multiculturales del estado español, contribuye a la unidad de la nación a la vez que bloquea la identidad específicamente andaluza del flamenco. Desde la reflexión de la instrumentalización política del flamenco, Moreno afirma que el flamenco es una cultura local y global, ya que se configura como un importante marcador cultural andaluz que reconoce la raíz mestiza e intercultural. Es un fenómeno que permite expresar los sentimientos y experiencias de clases gitanas y no gitanas. Debido a su funcionalidad social viva (enraizamiento cultural) para la expresión de valores, y no tanto a la mercantilización, ha tenido una proyección y acogida universal.

El 3 julio 1999, el Boja publicó el decreto por el que se declaraban bienes de interés cultural, con la categoría de Patrimonio Documental, los registros sonoros de la Niña de los Peines (Cruces, 2002, p. 164), lo cual supuso una materialización de la política patrimonial en relación al flamenco. Sin embargo, «la exclusiva consideración del flamenco como un género artístico supone seleccionar una significación cultural de calado mucho más hondo» (ibidem). Desde esta mirada el flamenco es un estilo artístico y una práctica tradicional con su historia propia, sin embargo, también es una realidad viva, una práctica funcional que persiste y se mantiene en un lugar central de la cultura andaluza. En relación a los instrumentos de la legislación sobre Patrimonio, la autora propone considerarlo expresión cultural completa, y para facilitar su clasificación en base a las claves administrativas, agrupa el flamenco en apartados fácilmente clasificables: (i) patrimonio material flamenco; (ii) expresiones músico-orales y plásticas del flamenco; (iii) espacios, prácticas y rituales; (iv) saberes y significaciones.

El flamenco ha sido reconocido como patrimonio cultural inmaterial de la humanidad por sus raíces culturales y etnológicas; muestra la diversidad cultural y representa una identidad; es tradicional a la par que está vivo; es la manifestación de un colectivo social; su transmisión es oral; y tiene significados y funciones psicosociales. Grötsch y De las Heras (2010), añaden 
(C) Servicio de Publicaciones de la Universidad de Murcia

http://revistas.um.es/flamenco

un componente emocional al patrimonio, ya que considera el cuerpo humano, como el principal instrumento para su ejecución o encarnación.

Una vez expuestas, a grandes rasgos, las dos corrientes de pensamiento, es posible describir los resultados del estudio que sitúa al flamenco en un lugar intermedio entre el arte y la cultura. Para ello, se parte de una mirada completa y no excluyente, que descubre en el flamenco elementos artísticos y culturales. A lo largo de cinco años de estudio doctoral específico sobre este fenómeno, y partiendo del marco teórico ya descrito, la figura 1 resume los elementos del flamenco, descrito en un sentido artístico y cultural.

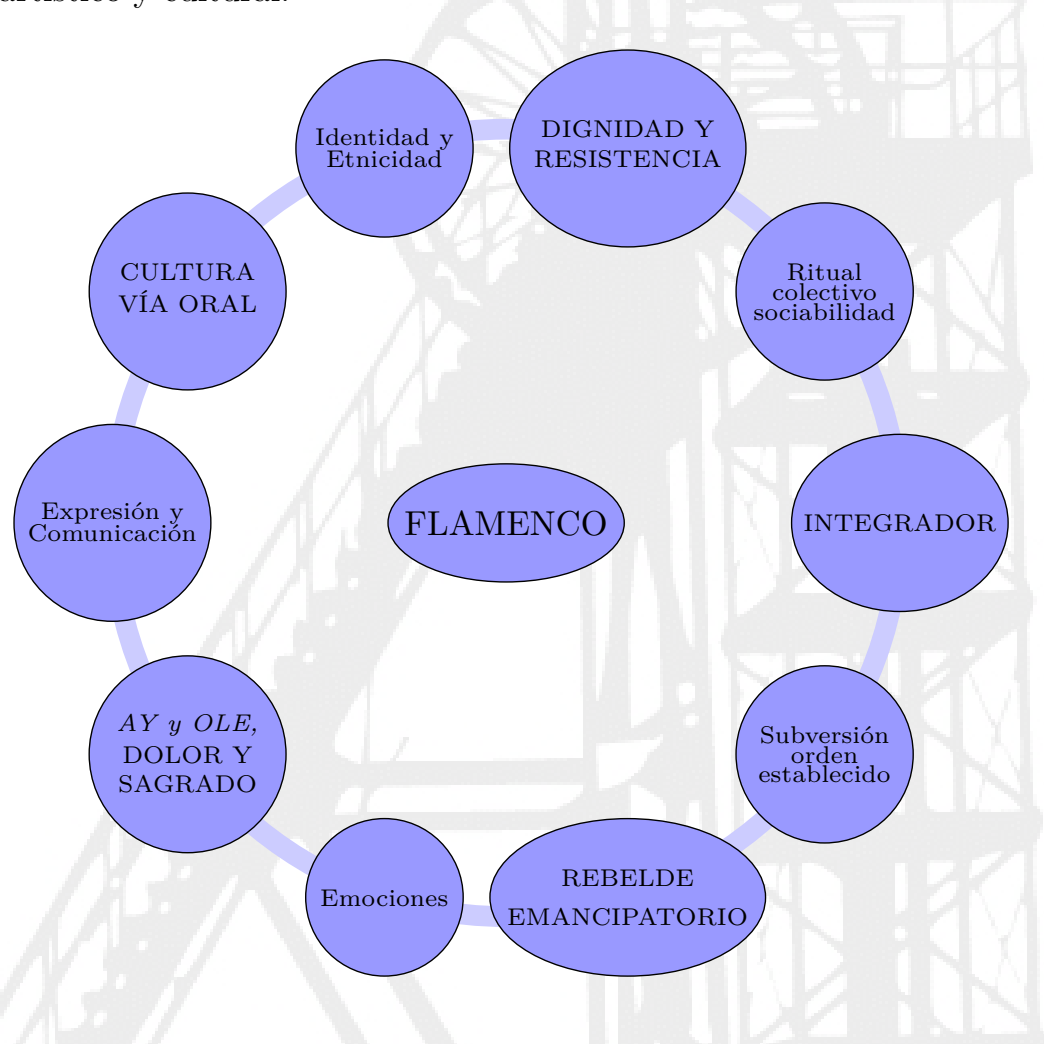

Figura 1. Elementos del flamenco (elaboración propia)

\section{Bases de la intervención social y educativa}

El objeto central de la investigación, es mostrar como el flamenco puede ser una herramienta de intervención social y educativa de éxito. Antes de continuar, nos detendremos a analizar en qué consisten ambas acciones.

La intervención social es aquella «actividad que se realiza de manera formal, intenta responder a necesidades sociales e incidir en la interacción de las personas, aspirando a su legitimación social»(Fantova, 2008, p. 149) La necesidad a la que se da respuesta es la interacción, esto es, la relación entre la autonomía personal y la integración comunitaria (Fantova, 2008). Es una actividad básica en el desarrollo de disciplinas como el Trabajo o la Educación Social (ibidem). La Psicología también trabaja mediante intervención, aunque emplea el término intervención psicosocial que podemos definir, siguiendo a Nelson y Prillenltensky, como «procesos intencionalmente 
diseñados para influir sobre el bienestar de la población por medio de cambios de valores, políticas, programas, distribución de recursos, diferenciales de poder y normas culturales» (Carnacea y Lozano, 2011, p. 188). En definitiva, la intervención social pretende que se dé un cambio para llegar al equilibrio, de modo que hay que entender la sociedad como un sistema social, formada por elementos e instituciones que la mantienen. No pretende la transformación del sistema sino la transformación de algunos elementos (personas, grupos, instituciones...), disfuncionales que deben ser estudiados y tratados para que se vuelvan funcionales (Montenegro, 2001).

La intervención social es producto del descontento social y de un deseo de cambio, puede darse como un proceso dirigido en el que será importante el conocimiento, o como un proceso participativo que dependerá de la acción colectiva como motor de transformación. Las intervenciones dirigidas no buscan un cambio completo de la sociedad, sino una mera reforma en la que ajustar las partes disfuncionales (Montenegro, 2001). Por el contrario, la intervención participativa sitúa como protagonista del proceso al grupo afectado. El cambio social deseable para este tipo de intervención, es la transformación de situaciones de injusticia estructural y asimetrías de poder, realizando intervenciones a escala micro para alcanzar un impacto mayor.

La intervención social tiene lugar al amparo de las metodologías desarrolladas por diferentes disciplinas, que ofrecen el marco y las técnicas para llevar a cabo la acción directa con las personas. La metodología no es solo la organización de unos pasos o instrumentos, sino que cuenta con una visión totalizadora e integradora que busca incidir en la estructura de los hechos objeto de intervención. Por tanto, hasta llegar a la práctica, el proceso metodológico es ascendente, supone una acumulación de conocimientos y utiliza diferentes técnicas para operar (Kisnerman, 1998).

Otra de las dimensiones en las que el flamenco nos permite intervenir con éxito, es la educativa, y para describirla, se parte de una mirada muy específica impulsada por Paulo Freire (1996), que la entiende como «praxis, reflexión y acción del hombre sobre el mundo para transformarlo» (p.1). Toda acción educativa parte de un concepto de lo humano y del mundo, es por ello que no hay una educación neutra, y tampoco puede ser al margen de la sociedad. Más bien, debe estar en contacto con el entorno y recoger las expectativas, sentimientos, vivencias y problemas de las personas.

La educación para Freire (1996) es un canto de amor, de coraje hacia la realidad, que no teme y que más bien busca transformar, con espíritu comprometido y fraternal. Por ello la educación es diálogo, comunicación entre los hombres y no se da en el vacío sino en escenarios sociales concretos. Es la herramienta más poderosa de trasformación social, es arma de lucha contra el atraso y la pobreza, es una vivencia constante de experiencias mutuas entre el educador y el educando, que dan pie a la educación concientizadora.

Entendiendo la educación de este modo, es posible entender también como el flamenco, es una herramienta que nos facilite esa liberación y transformación de la que habla Freire (1996), ya que son varios los elementos que conforman el flamenco, que están muy relacionados con la definición de educación dada. Nos referimos a la sociabilidad, al acto social en el que es posible el flamenco, el papel que cobran la expresión y la comunicación, y la propia identidad de rebeldía y denuncia que hay en su raíz más honda. De este modo, el flamenco no solo como elemento cultural, sino como elemento artístico, puede favorecer el trabajo educativo y social.

Para comprender la relación entre flamenco y educación, es importante también analizar la dimensión del arte en relación a la intervención socioeducativa. Si analizamos la práctica directa de la intervención desde el Trabajo Social, la Educación Social o la Psicología, detectamos que las técnicas relacionadas con el arte se emplean a menudo como elementos mediadores para la educación o contra la exclusión. Se trata de técnicas culturales y artísticas que los profesionales emplean en contextos sociales y comunitarios sin conocimiento ni planificación específica, contando únicamente con su propia creatividad (Moreno, 2010). La intención es utilizar el arte como 
(C) Servicio de Publicaciones de la Universidad de Murcia

http://revistas.um.es/flamenco

mediador, como una herramienta que permite al profesional fomentar la autonomía y la inclusión social de las personas (ibidem). La experiencia artística descubre la propia identidad del sujeto y su imaginario, de manera que accede al plano simbólico. Facilita la comprensión del "yo" y el "mundo" como representaciones moldeables dentro de un marco social y cultural. Según Moreno (2010), «nadie puede salir de una situación de exclusión social si antes no ha sido capaz de imaginarse de otra manera, de representárselo, de proyectarse hacia el futuro»(p. 2). De modo que el arte puede ser vehículo para una representación diferente de la persona, como una proyección hacia donde se dirige su propio cambio.

Desde disciplinas como la filosofía, la psicología o la educación, diferentes autores piensan sobre la relación entre el arte y la intervención social. El filósofo Nelson Goodman (1906-1998) mantiene que el arte permite entender y cambiar la representación de la sociedad que nos hacemos, dando pie a una relación entre lo sentido y lo vivido. Desde la psicología, autores como Sigmund Freud (1856-1939), Carl G. Jung (1875-1961), Jean Piaget (1896-1980), María Cristina Rojas o Susana Sternbach, han trabajado las relaciones entre el desarrollo humano y el arte, destacando unos el desarrollo intelectual, y otros el emocional según sus corrientes de pensamiento. Para el psicólogo gestáltico Arnheim, el arte ayuda a situarse en el mundo y afirma que es uno de los instrumentos más poderosos para la realización de la vida (Moreno, 2010)

Moreno (2010) plantea hasta cuatro modelos diferentes de intervención. El modelo academicista pone el acento en la representación y no tiene en cuenta al sujeto. El expresionista promueve el desarrollo de la expresión y la creatividad a través del arte. El modelo de alfabetización visual entiende que el arte y las representaciones visuales funcionan como un lenguaje y que la Educación Artística ha de consistir en aprender sus reglas como si se tratara de una gramática. Por último, el modelo que denomina "ecuación para la comprensión de la cultura visual" trabaja la capacidad de analizar e interpretar de forma crítica el mundo desde las representaciones visuales, sin atender a la producción (Moreno, 2010, p. 4). El flamenco, puede operar en cualquiera de los cuatro modelos, aunque en las experiencias investigadas, se desarrolla con más coherencia en los modelos dos y tres descritos por Moreno.

Otra forma de intervención que ha alcanzado bastante popularidad es la arteterapia, entendida como una forma de psicoterapia a través del arte que nace después de la $2^{\text {a }}$ Guerra Mundial en EEUU Alemania e Inglaterra, hasta los 90 no se implantó en España. A diferencia de las terapias basadas en la palabra, en arteterapia la persona se expresa mediante símbolos y producciones artísticas a partir de las cuales reflexiona sobre sus conflictos y dificultades, dando más importancia al proceso que al resultado. Se puede realizar en grupo o individual y no tiene límite en edad o problemática, cobra importancia el proceso de transformación personal que se facilita con las actividades (Moreno, 2010).

En el ámbito educativo, De las Heras (2013) mantiene que la Educación Artística permite un desarrollo integral y emocional a través de la inclusión del arte en procesos educativos. Se trata de una herramienta pedagógica de gran calado ya que, a través de ella, el alumnado desarrolla sus recursos cognitivos, perceptivos, valoración crítica y expresión. Para ello, la educación artística ha de ser crítica e ir más allá de las habilidades técnicas del alumno, debe ser una reflexión a partir de la creación colectiva del alumnado. Considera, además, que la danza debería ser una materia a desarrollar en la educación, así como un tema de investigación y la describe como aquella expresión de sentimientos que tiene lugar a través del cuerpo, el espacio y el tiempo y que, a su vez, permite la socialización y el intercambio de roles.

Dicho lo anterior, consideramos que el flamenco entendido como fenómeno artístico, según defienden autores como Steingress o Lavaur, puede ser empleado como herramienta en la intervención socioeducativa, desarrollada a través del arte.

Respecto a la educación, también es necesario abordar la cuestión de la Innovación Educativa 
como aliada en un escenario que, cada curso se torna más complejo, por la propia evolución de la sociedad, a la cual la educación debe seguir el ritmo sin dilación.

Como se afirma en el portal de la Junta de Andalucía, en el apartado Innovación educativa, «la innovación en educación es un proceso permanente, original e intencional de búsqueda de mejora de la calidad educativa y de los aprendizajes del alumnado; su relación con el uso de las tecnologías está supeditada a esa mejora e incluye aspectos estructurales que incumben a la organización de los centros, la dinámica de información y comunicación y, sobre todo, los procesos de enseñanza-aprendizaje ` ${ }^{2}$ A este respecto, Francesc Pedró, director del Instituto Internacional de la Unesco para la Educación Superior en América Latina y el Caribe (IESALC), señala que «es innovación si añade valor al aprendizaje»?

Siguiendo las claves dadas por la Junta de Andalucía, la innovación es un campo abierto a múltiples posibilidades, que a priori se centran en las nuevas tecnologías, pero también deben atender a la parte humana y vivencial de la persona en un entorno que no siempre es el más agradable, como por ejemplo el que hemos vivido este año debido a la crisis sanitaria. Obligada toda la comunidad educativa, a alterar sus costumbres, formas de relacionarse y de operar en los centros, ha sido necesario innovar para mejorar las formas tradicionales con el fin de prevenir la enfermedad y mantener los niveles de relación y vínculo social necesario para el proceso de enseñanza-aprendizaje.

Sirva esta situación como ejemplo para la reflexión que sigue. Si se entiende la innovación como algo más allá del equipamiento TIC en los centros, será posible crear nuevas realidades en el espacio educativo que hagan más rico el proceso de enseñanza - aprendizaje. Todo ello mediante el cuidado del vínculo y la afectividad, bajo el respeto al derecho de la educación, teniendo en cuenta los múltiples agentes participantes y con apertura al entorno social.

En definitiva, la innovación provoca un cambio en la realidad educativa, que haga mejorar las claves de la enseñanza, y provoquen la actualización necesaria para acompasar el trabajo en los centros, con la vida real. Una de las claves del éxito es tener en cuenta el contexto, para adaptar el trabajo a las personas, evitar obstáculos y potenciar las fortalezas de la comunidad. Es preciso que la escuela se configure como un espacio abierto, que permita la entrada y participación de las familias y la comunidad en el proceso educativo. Hargreaves (2003) plantea que el cambio en educación está relacionado con la atención a los aspectos racionales y emocionales de este cambio, así como con su expansión al resto de la comunidad. Según el autor, es preciso aunar compromisos y trabajar de manera conjunta entre comunidad e instituciones (Sanchiz, 2009).

Partiendo de la información anterior, teniendo en cuenta las claves educativas dadas por Freire (1996), junto con las posibilidades que ofrece la innovación para mejorar el proceso enseñanzaaprendizaje. Es posible plantear la aproximación del flamenco al entorno educativa como experiencia innovadora, que nos comunique con el entorno cultural que se habita, favorecer la expresión y la comunicación, la sociabilidad, el diálogo y en última instancia la transformación.

Para fijar la relación establecida entre educación y flamenco en las intervenciones estudiadas, se muestra el siguiente gráfico (figura 2), en el que se cruzan los aspectos de la educación destacados por Freire (1996), con el análisis teórico del flamenco presentado en el apartado anterior.

\section{Metodología}

La investigación doctoral que enmarca los resultados descritos en el presente artículo, se aborda desde el método etnográfico, precedido de una revisión bibliográfica y documental de fuentes

\footnotetext{
${ }^{2}$ Recuperado de: https://www.juntadeandalucia.es/educacion/portals/web/ced/innovacion-educativa.

${ }^{3}$ Ibidem.
} 
(C) Servicio de Publicaciones de la Universidad de Murcia

http://revistas.um.es/flamenco

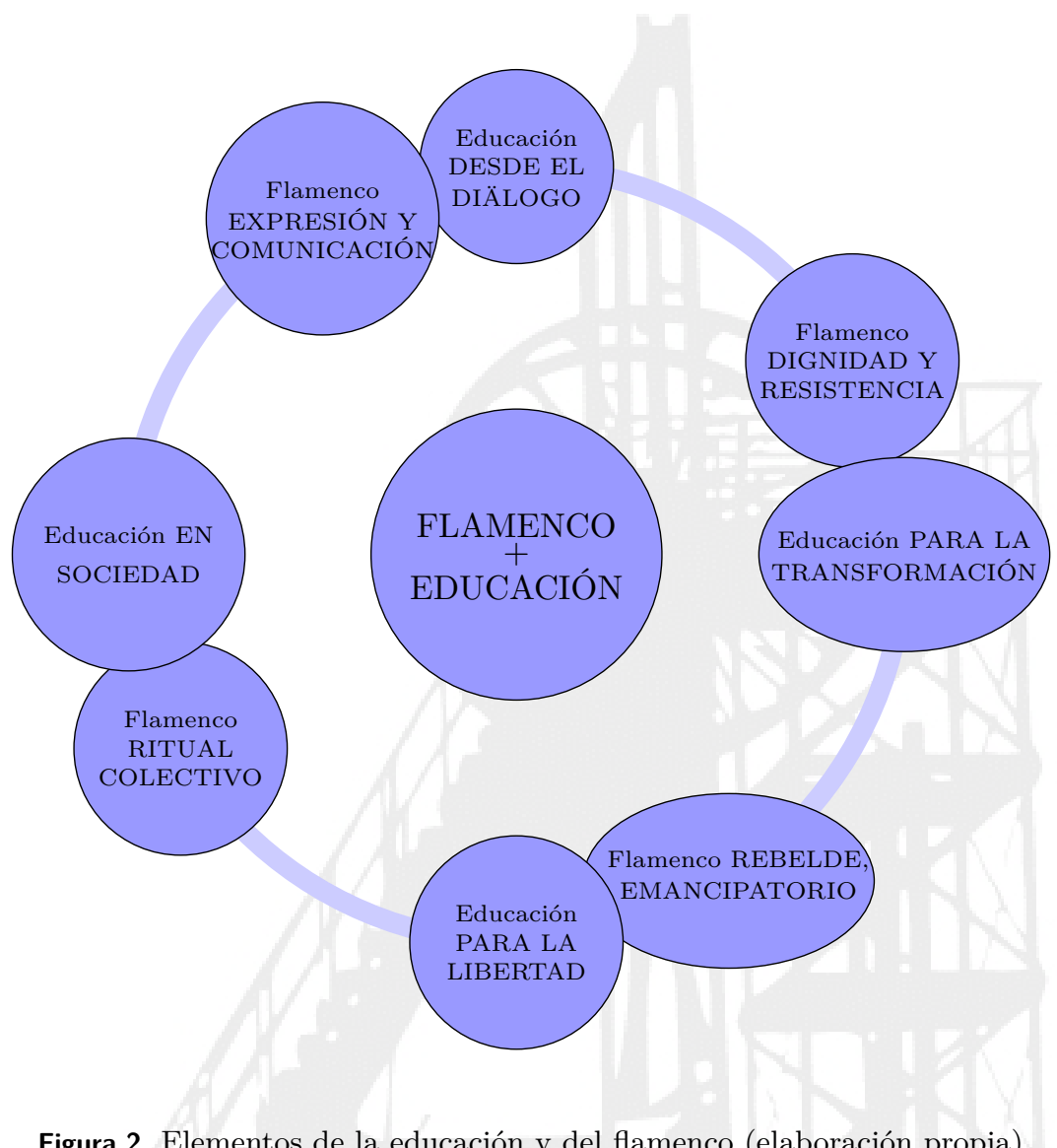

Figura 2. Elementos de la educación y del flamenco (elaboración propia)

primarias y secundarias que permiten iniciar el trabajo de campo desde una perspectiva más próxima al objeto de estudio.

\section{3.1 Revisión documental}

Se realiza una revisión documental de fuentes primarias derivadas de la Realidad Social estudiada, así como fuentes secundarias: textos, artículos científicos, conferencias, noticias de prensa que se hayan escrito sobre el Objeto de Estudio, o que puedan arrojar luz a la hipótesis planteada y que permitan la aproximación al campo con una información previa necesaria para comprender y analizar las situaciones reales través de la exploración de los principales autores y obras descritas en el marco teórico, que son referencia de la investigación. En relación al estudio del flamenco como fenómeno artístico y cultural analizaremos las principales obras de Lavaur, Steingress, Whasabaug, Aix, Cruces, Mandly y Moreno. Dentro del marco de la intervención social a través del arte, se revisa la producción de Grötsch y de las Heras, así como autores de referencia en Psicología, Trabajo social o Educación: Jung, Piaget, Klein, Ander- Egg y Freire entre otros. Por último, se atiende a las publicaciones que de manera específica contemplan la aplicación del flamenco en la intervención social. 
Revista de Investigación sobre Flamenco "La madrugá" n. ${ }^{\circ}$ 18, diciembre 2021 , ISSN $1989-6042$

\section{3.2 Método etnográfico}

La etnografía es el método desarrollado por la disciplina antropológica. Se materializa en el trabajo de campo y ofrece varias herramientas para acceder a la información, de las cuales se utilizan la observación y las entrevistas en profundidad a informantes clave para el estudio.

La etnografía promueve un contacto directo y prolongado en el campo, es flexible en sí misma y emplea varias fuentes en la investigación, todo ello permite una óptima triangulación de la información para su comparación. Según Velasco y Díaz (2006), «su principal característica sería que el etnógrafo participa [...] en la vida diaria de las personas durante un período de tiempo, observando qué sucede, escuchando que se dice, haciendo preguntas» (p. 15). En ese sentido, la calidad de los datos obtenidos está directamente relacionada con la aproximación del investigador o investigadora al tema que nos ocupa, a los espacios, a los agentes participantes y sus interacciones, procurando el conocimiento directo de primera mano y reduciendo al mínimo las personas que intermedian.

\subsubsection{Aplicación del enfoque de género en la etnografía}

La propia investigación está diseñada de manera sensible al género por lo que las técnicas aplicadas permiten recopilar información orientada a clarificar la cuestión del género en relación al objeto de estudio, así como su categorización en base a un sistema de indicadores, durante el proceso de análisis de los datos de campo. Para ello se aplica la transversal de género empleando de manera complementaria sus dos enfoques: el GED y el MED los cuales, a partir del pensamiento feminista aportan una batería de herramientas de aplicación y evaluación de la implementación del género en el objeto de estudio. Se utilizan los indicadores de género en el proceso de análisis de los datos, para contrastar toda la información recopilada a través de otras herramientas. Los indicadores se diseñan de manera previa a la inmersión en campo en relación a las unidades de observación descritas, pese a ello pueden ser modificables en el transcurso de la investigación. Como señala Dávila (2000), «un indicador es un señalador [...] una medida, un número, un hecho, una opinión o una percepción que señala una situación o condición específica y que mide cambios en esa situación o condición a través del tiempo» (p. 10).

Murguialday, Vázquez y González (2008) señalan que el indicador de género «mide las consecuencias positivas y negativas que ha tenido una intervención [...] en las relaciones de género» (p. 10). Los indicadores se aplican en el análisis de los datos obtenidos con el método etnográfico, medirán aspectos de proceso y resultados, tanto cuantitativos como cualitativos a lo largo de todo el trabajo de campo y serán una referencia para contrastar y categorizar información ${ }^{4}$.

\subsection{La inmersión en el campo}

El trabajo de campo comienza con la inmersión de la investigadora en el contexto del objeto de estudio, que va más allá de la simple presencia en el campo y requiere una negociación los agentes de cada unidad de observación de manera que acepten la presencia de una persona ajena con un fin académico. En los lugares públicos se desarrollan rituales de interacción en los que la observación puede provocar alteraciones en el propio ritual, por tanto, se ha negociar la presencia y participación de manera que no se ponga en riesgo el objeto de estudio. Según Hammersley y Atkinson (1994), «los lugares públicos pueden caracterizarse por un tipo de interacción social que hace referencia a lo que Goffman denomina desatención civil» (p. 72). En este sentido la protección de la investigadora en el campo, a través del padrinazgo por relaciones personales y de trabajo, está relacionada con la calidad de la información que se obtiene. Las primeras

\footnotetext{
${ }^{4}$ Se elaborarán los indicadores de género objetivamente verificable, adaptados a cada observación de campo.
} 
(C) Servicio de Publicaciones de la Universidad de Murcia

http://revistas.um.es/flamenco

entradas al campo se apoyan en relaciones ya creadas previamente entre la investigadora y los actores, así como en relaciones creadas con personas de referencia, que a menudo adoptan un rol de padrinazgo respecto a la investigadora, a la que acompañan durante su recorrido por el campo (Velasco y Díaz 2006, p. 104). Para asegurar la calidad de la información que se obtiene durante el trabajo de campo, de manera que no altere las conductas y comportamientos habituales del grupo (Rubio y Varas, 1997, p. 456), la presencia en el campo se ha de consolidar muy lentamente, para que no perturbe el libre desarrollo del objeto de estudio, ni suponga una intromisión a su espacio de actuación: ha de ser una intrusa y recíproca al mismo tiempo. La sistematización de los datos de campo ha de ser rigurosa y complementada con medio audiovisuales que den lugar a la producción documental de la información.

\section{3.4 La observación}

La técnica de la observación a menudo facilita la aproximación al objeto de estudio. Responde a una planificación previa en la que se identifican y seleccionan las unidades de observación más importantes. Nos obliga a establecer relaciones igualitarias con las personas durante el Trabajo de Campo, puesto que «la información se intercambia a modo de comentarios. Por otra parte, requiere el aprendizaje y seguimiento de las reglas del grupo» (Velasco y Díaz, 2006, p. 25). La aplicación del relativismo metodológico es fundamental en la observación participante, de manera que el campo devuelva informaciones objetivas. En este sentido Lévi Strauss (1969) señalaba que «el antropólogo hace algo más que acallar sus sentimientos: elabora nuevas categorías mentales, contribuye a introducir nociones de espacio y tiempo, de oposición y contradicción, extrañas al pensamiento tradicional» (p. 327). En la investigación se atiende a tres principios que aseguran el desarrollo científico de la observación, según König (1973; cit. por Velasco y Díaz, 2006, p. 23). La constancia, el control de la observación mediante instrumentos acordes a la investigación, y por último la orientación del diseño teórico de la investigación.

Rubio y Varas (1997, p. 456) definen tres tipos de observación, en base a los niveles de participación, a la sistematización de la información recopilada y al tipo de información. En esta investigación prevalece la observación participante y, con la observación no sistematizada más flexible y abierta, que permite aportar múltiples datos en las primeras inmersiones en el campo o en investigaciones como la que nos ocupa, cuyo objeto de estudio es un fenómeno sobre el que aún no hay orientaciones, al tiempo que ayuda a definir el diseño de la investigación. A medida que se realizan más inmersiones en el campo, la observación va adquiriendo un mayor grado de estructuración previa que permite la concentración en un foco de atención, hasta el punto de convertirse en Observación Sistematizada, para la cual se diseña un listado de categorías de observación que permite la clasificación y cuantificación de la información. En esta estructuración de la observación, se incorpora como categoría transversal el enfoque de género, que permite el análisis de la información obtenida, desde esta categoría. Por último, atendiendo al tipo de información que se pretende obtener, se trabaja una observación cualitativa y cuantitativa. A pesar, de que en el método etnográfico prima la información cualitativa, es relevante combinarla con datos cuantitativos mediante la observación, por ejemplo, del número y tiempo de las personas participantes en las intervenciones, desagregando los datos por sexo, además de estudiar cualitativamente, el contenido de las interacciones dadas diferenciando entre hombres y mujeres. En la presente investigación, se utiliza fundamentalmente una observación participante, no sistematizada y cualitativa; pese a ello es un continuo que adopta una forma u otra en base al contexto y las relaciones con el campo, en la cual tienen influencia las características adscritas y descritas de la investigadora en el campo. Durante el desarrollo de la Observación se procederá al registro documental de la información mediante la grabación, las fotografías y el diario de campo, 
siendo este una herramienta fundamental en la recopilación diaria de todos los movimientos e informaciones que se llevan a cabo.

\subsubsection{Unidades de observación}

Para que la observación sistematizada sea posible, se definen aspectos en los que se va a poner mayor atención, esto es las unidades o categorías de observación «son conjuntos de conductas que conforman un constructo [...] una abstracción que alberga toda una serie de manifestaciones conductuales» (Rubio y Varas 1997, p. 456). En este caso, se trata de categorías de forma empírica, es decir se proponen partiendo de la observación de la realidad inicial y son susceptibles de cambio o ampliación, según se den los acontecimientos.

Para que la observación participante tenga éxito, se promoverá alcanzar un grado de sociabilidad mínimo con los actores sociales y considerar en todo momento que la investigación es un continuum y no solo es posible cuando se activa la grabadora o cuando se realiza la observación, sino que más bien se lleva a cabo de manera constante y en un trabajo de relación continuada con nuestro objeto de estudio (García del Villar Balón, 2014). Es por ello que los espacios de sociabilidad, aquellos que nos son exclusivos de la investigación, son claves para la recogida de información de manera informal. Así mismo, para llevar a cabo un óptimo trabajo de campo, se debe desarrollar la empatía con las personas que se observan y entrevistan. La confidencialidad es un principio que debe cumplirse por parte de la investigadora, asegurándola al inicio del trabajo y las entrevistas, a todos los miembros implicados.

Es importante diferenciar en nuestro diario de campo, qué información ha sido recogida de manera formal y qué información de manera informal, puesto que debe ser sistematizada atendiendo a este criterio. Entendemos por formal, aquella información que es recopilada durante una entrevista semiestructurada, y por informal todos los datos obtenidos durante la observación participante, esté o no planificada.

\section{3.5 La entrevista}

Otra herramienta fundamental empleada en el trabajo de campo es la entrevista, entendida como un encuentro hablado entre dos individuos que comporta interacciones verbales y no verbales, (Pope, 1979), en ese sentido se encuentra muy próxima a la observación participante ya que en ambos casos se ha de tener en cuenta el contexto y la influencia de la investigadora. Se complementa además con la observación, en el sentido en que pueden confirmarse o no determinadas informaciones comparando ambas herramientas. La observación en estas situaciones, así como el grado de empatía e inmersión que hayamos desarrollado con el colectivo, nos permitirá identificar los agentes clave a los que realizar las Entrevistas en profundidad. Señalar que en el trabajo de campo se pueden emplear diferentes modalidades de entrevistas, según la clasificación de Rubio y Varas (1997, p. 409). Atendiendo al objetivo de la entrevista, esta puede ser clínica, de orientación o de investigación. Por otra parte, según la estructuración y directividad puede ser una entrevista estructurada (directiva) o semiestructurada (abierta), esta última parte de un guion de temas a tratar en la conversación, que simplemente es guiada por la investigadora, centrando el peso y protagonismo a la persona entrevistada, el papel de la investigadora debe dar confianza y permitir el discurso fluido que hile unos temas con otros. En base a esta clasificación, optaremos por desarrollar la entrevista de investigación semiestructurada, sin embargo, es posible que en ciertos casos sea necesario trabajar la entrevista estructurada porque así lo requiera el contexto y la persona entrevistada.

La selección de informantes suele ser una tarea complicada, para la cual hay que tener en cuenta que no es decisión única del investigador, más bien «se trata de una elección relativa, puesto que el etnógrafo también es elegido por los informantes clave» (Velasco y Díaz, 2006, p. 106). El 
(C) Servicio de Publicaciones de la Universidad de Murcia

http://revistas.um.es/flamenco

criterio que debemos aplicar para la selección de personas a las que entrevistar, es la Pertinencia, para el curso de nuestra investigación. Es decir, se elegirá «a personas que sean significativas en relación a los objetivos de la investigación y a la población a la que esta se refiere» (Rubio y Varas, 1997, p. 429). Hoffman aconseja realizar la selección de informantes mediante las recomendaciones de los propios informantes seleccionados a priori por la investigadora, sin embargo, esto se ha de gestionar con sumo cuidado, ya que puede provocar un sesgo en la información. El tipo de informante al que se entrevista condiciona el desarrollo de un tipo de entrevista u otro, puesto que la información que se obtiene es diferente y requiere por ello un tratamiento distinto. Existe una división fundamental entre informantes, una misma persona puede pasar de una categoría a otra según sea el desarrollo de la investigación, que está sujeto a una multiplicidad de variables internas y externas que condicionan la posición y situación del informante. Siguiendo a Rubio y Varas (1997), establecemos una división de tres tipos de informantes: (i) los informantes directos son aquellas personas que están implicadas directamente en el objeto de estudio; (ii) los informantes indirectos son aquellos sujetos que participan del objeto de estudio de manera colateral; (iii) finalmente, los informantes clave son aquellas otras personas que disponen de información privilegiada sobre el objeto de estudio, sin ser integrantes del núcleo central.

En relación al ámbito educativo, se ha llevado a cabo una investigación, siguiendo la metodología descrita, para conocer en profundidad las iniciativas recogidas en la tabla 1.

\begin{tabular}{|c|c|c|c|}
\hline EXPERIENCIA & CENTRO O PROGRAMA & AÑO & PARTICIPANTES \\
\hline \multirow{5}{*}{$\begin{array}{l}\text { EXPERIENCIA A: } \\
\text { Ponte flamenca }\end{array}$} & IES Levante (Algeciras) & 2015 & Alumnado FP y profesorado \\
\hline & IES Galileo Galilei (Córdoba) & 2016 & Alumnado FP \\
\hline & IES Averrores (Córdoba) & 2017 & Alumnado ESO \\
\hline & CEP Córdoba & 2018 & Profesorado \\
\hline & IES Cornelio Balbo (Cádiz) & 2000 & Profesorado \\
\hline $\begin{array}{l}\text { EXPERIENCIA B: } \\
\text { Proyecto Innovación } \\
\text { Educativa }\end{array}$ & $\begin{array}{l}\text { Programa Vivir y Sentir el } \\
\text { Patrimonio, proyecto de } \\
\text { innovación educativa a través } \\
\text { del Flamenco \#Levanterías, } \\
\text { IES Levante (Algeciras) }\end{array}$ & $\begin{array}{l}2020- \\
2021\end{array}$ & Comunidad Educativa \\
\hline \multirow{3}{*}{$\begin{array}{c}\text { EXPERIENCIA C: } \\
\text { Premios Flamenco en } \\
\text { en Aula } \\
\end{array}$} & IES García Lorca (Algeciras) & 2020 & \multirow{3}{*}{ Comunidad Educativa } \\
\hline & IES Jacaranda (Brenes] & 2020 & \\
\hline & IES Las Lagunas (Mijas) & 2020 & \\
\hline \multirow{3}{*}{$\begin{array}{l}\text { EXPERIENCIA D: } \\
\text { Intervenciones en } \\
\text { espacios educativos } \\
\text { no formales. }\end{array}$} & $\begin{array}{l}\text { Fundación Alcalá (Sevilla y } \\
\text { Jerez) }\end{array}$ & 2020 & $\begin{array}{l}\text { Residentes del barrio } 3.000 \\
\text { viviendas de Sevilla. Menores } \\
\text { y sus familias }\end{array}$ \\
\hline & Ole con Ole & 2015 & $\begin{array}{l}\text { Educación para el Desarrollo } \\
\text { en colaboración con la ONGD } \\
\text { CIC Batá }\end{array}$ \\
\hline & Flamenco Inclusivo & 2018 & $\begin{array}{l}\text { Personas con diversidad } \\
\text { funcional adscritas a ONCE y } \\
\text { Asociación Síndrome de } \\
\text { Down Sevilla }\end{array}$ \\
\hline
\end{tabular}

Tabla 1. Intervención educativa a través del flamenco: experiencias analizadas 
Revista de Investigación sobre Flamenco "La madrugá"

n. ${ }^{\circ}$ 18, diciembre 2021 , ISSN $1989-6042$

\section{Flamenco y educación: experiencias de éxito}

Se describen a continuación las principales claves de las experiencias estudiadas, que guardan una relación directa con los aspectos más relevantes del flamenco como elemento social y cultural, según el marco teórico descrito al comienzo del artículo.

\section{4.1 Experiencia A: Ponte flamenca}

Esta experiencia ha sido estudiada en profundidad, tanto en tiempo, como en forma. Ello ha permitido obtener una mirada más completa de su desarrollo, que ha tenido lugar en varios centros educativos de la Comunidad Autónoma Andaluza. En un período temporal amplio, desde septiembre de 2015 hasta el pasado año 2020. Con la presencia de alumnado de diferentes etapas educativas (FP y ESO), así como profesorado. También es relevante como se ha desarrollado a través de diferentes formatos: Talleres educativos para el alumnado y profesorado, Formación en el Centro de Profesorado de Córdoba, así como Grupos de Trabajo durante el curso escolar.

La esencia de esta intervención, es la flexibilidad y la colectividad. Mediante el trabajo grupal abierto al cambio, es posible adaptarse a las diferentes necesidades o temáticas solicitadas por el grupo, que pueden versar sobre la transmisión de contenidos, la perspectiva de género, la cohesión grupal, la confianza y el conocimiento de los participantes, el trabajo emocional, la danza y la expresión corporal o bien el trabajo en torno a las letras flamencas y la expresión verbal.

El flamenco es el hilo conductor de esta intervención y, por tanto, elemento transversal a todo el trabajo. La propuesta emplea herramientas propias de la intervención socioeducativa como la dinámica de grupos, el teatro social, la creación colectiva... y lo hace apoyándose en los elementos que conforman el flamenco, que son cante, baile, guitarras compás, letras, música y expresión cultural.

\subsection{Experiencia B: Proyecto innovación educativa}

Esta iniciativa ha sido desarrollada en el marco del Programa "Vivir y Sentir el Patrimonio", como un Proyecto de Innovación educativa a través del Flamenco. Su nombre es \#Levanterías y es un proyecto de flamenco puesto en marcha por el IES Levante de Algeciras. Ha sido impulsada por un equipo de docentes de diferentes áreas y etapas educativas, durante el curso 2020/2021, con el objetivo de aproximar el flamenco a las aulas como una herramienta de innovación educativa, que permita mejorar el proceso de enseñanza-aprendizaje. El proyecto ha desarrollado acciones en torno a la figura de Paco de Lucía, con la intención de resignificar al genio flamenco, a la vez que a la ciudad en la que nace, como un lugar de gran riqueza patrimonial. En el proyecto ha participado toda la comunidad educativa, las dificultades impuestas por el cumplimiento del protocolo Covid en el centro, han permitido un mayor acceso a las actividades, a través de la difusión por medios audiovisuales.

La esencia de esta intervención, es el amor y la afición del profesorado que la promueve, tanto por el flamenco, como por una educación pública, innovadora y de calidad. Todas las acciones parten de un conocimiento muy básico del profesorado sobre flamenco, que se ha visto acompañado por artistas locales a lo largo del curso.

El flamenco es el elemento central, a través del cual vivir el patrimonio local. Cobra un papel fundamental para repensar ideas y prejuicios más que viciados, sobre una región en la que pesa demasiado el narcotráfico y la delincuencia. La vivencia de este arte, a través de Paco de Lucía, una de las figuras más representativas de la etapa de Flamenco Moderno, permite identificar claves positivas y poner en valor el entorno próximo, también como fuente de riqueza. 
(C) Servicio de Publicaciones de la Universidad de Murcia

http://revistas.um.es/flamenco

\section{4.3 Experiencia C: Premios flamenco en el aula}

Los Premios Flamenco en el Aula, reconocen el éxito del trabajo realizado en los centros a través de iniciativas como la experiencia descrita anteriormente, con la organización de un proyecto de innovación educativa enmarcado en el proyecto "Vivir y Sentir el Patrimonio". Es interesante destacar como cada proyecto parte de su contexto para crear la propuesta, ya sea Sevilla, Cádiz o Málaga, y como todos los lugares comparten un código cultural asociado al territorio Andaluz.

La esencia por tanto de cada proyecto, parte de las experiencias y recursos personales de la comunidad educativa (docentes o familias aficionadas, población de etnia gitana, alumnado aprendiz de guitarra, o baile flamenco). Ello sumado a una mirada realista al entorno en el que se encuentra ubicado el centro, que en cada caso va a dar una clave de trabajo sobre la que articular el proyecto.

El flamenco, una vez más se convierte en herramienta de innovación educativa, sale del aula de música y se hace presente en el resto de áreas formativas, para acompañar e innovar a alumnado y profesorado en nuevas formas de desarrollar el proceso enseñanza- aprendizaje.

\subsection{Experiencia D: Intervenciones en espacios educativos no formales}

Por último, el estudio de la intervención educativa en espacios no formales, a través del trabajo de profesionales que colaboran o forman parte de entidades sociales como ONCE, CIC Batá o Fundación Alalá. En todos los casos, el trabajo se realiza con población en situación de exclusión social, y precisamente de ahí, parte la intervención educativa, como una propuesta de transformación de la realidad social y personal de estos colectivos.

La esencia de estas propuestas, está relacionada con las bases del Trabajo y la Educación Social. Ambas provocan un giro en la realidad de las personas, para favorecer un cambio orientado a mejorar su realidad. En todos los casos, se emplea el flamenco como vehículo sobre el cual, ir abriendo ese camino hacia la evolución personal.

El uso del flamenco permite conectar con los colectivos en situación de vulnerabilidad, ya sea en el barrio de Triana en Sevilla, o en las Fabelas de Brasil. El flamenco canta el sufrimiento y la herida de una población mestiza, gitana, negra, judía, en definitiva, excluida durante siglos y que encuentra en este arte su medio de expresión. Ese lamento será el mismo en cualquier lugar del planeta que se haya dado una injusticia, y de este modo es posible la conexión desde la expresión cultural de los cantes de raíz. Y solo ocurre con estas músicas, que tienen esa doble dimensión cultural y artística, como señala Francisco Aix (2014).

Para entender el trabajo que realizan los profesionales de la educación formal o no formal a través del flamenco, es necesario entenderlo como un elemento cultural, y dese ahí, nos aproximamos a la tesis de Cristina Cruces, para dar respuesta a porqué surge y se desarrolla de manera específica en Andalucía. Lo hace como una forma de transmisión cultural, a través de la cual, el pueblo se expresa y se mantienen vivas sus expresiones, creencias y formas de relacionarse. «El flamenco nació para sobrevivir, el flamenco es vida, porque lo parieron supervivientes, en el momento en que se fosilice, morirá, porque se convertirá en una de esas culturas que nada tiene que ver con su $\mathrm{ADN} \gg\left(\mathrm{EP} 3 . \mathrm{AM}^{5}\right.$ ).

Desde esta mirada, el origen del Flamenco tiene lugar como una forma de resistencia a la injusticia que vivió el pueblo andaluz. Frente a las teorías habituales que lo sitúan como un fenómeno artístico que surge en el s. XVIII, Antonio Manuel aboga por una creación anterior que bebe de la historia, que se va gestando en el seno de un pueblo que sufre y lucha por sobrevivir, y

\footnotetext{
${ }^{5}$ Entrevista en profundidad 3. Antonio Manuel Rodríguez (25-03-20).
} 
no tanto por generación espontánea. En ese caso, se confunde la primera aparición del flamenco, con su origen:

\begin{abstract}
El grito se quedó ahí. Por eso se canta en andaluz. Clavado en los tuétanos del pueblo que no abdicó de sus raíces, a pesar de la persecución e intento de exterminio [...] no perdieron la memoria $[. .$.$] en un ejercicio sublime de resistencia, los que se quedaron siguieron convocando$ a la oración y otras prácticas espirituales propias del sufismo popular andalusí, con el grito y dolor incrustado en las entrañas. Ya no podían hacerlo en público ni en algarabía [...] pero lo hicieron en la oscuridad y en la lengua nueva que nació al mezclar la impuesta con las lenguas de los distintos (Rodríguez, 2018, p. 30).
\end{abstract}

\title{
5. Conclusiones
}

Adentrarse en las experiencias de intervención socioeducativa a través del flamenco, permite identificar las claves del éxito en las iniciativas, que deciden navegar con el flamenco como barco, por las aguas de la educación, para la transformación social y personal.

A partir del marco teórico que define el flamenco como elemento cultural y artístico, se identifican una serie de elementos presentes en esta música, sobre los cuales podemos apoyar la intervención educativa, de manera que el flamenco se convierte en un apoyo y un soporte para el proceso de enseñanza-aprendizaje:

- El flamenco es posible como forma ritual colectiva. La educación es necesariamente social.

- El flamenco es una forma de expresión y comunicación desde un código cultural particular. La Educación solo puede darse desde el diálogo con las personas.

- El flamenco mantiene una identidad cultural propia. La educación defiende la libertad de ser de las personas.

- El flamenco es un grito de rebeldía y denuncia de la injusticia. La educación es la herramienta más poderosa de transformación social.

Todos los elementos descritos están presentes en las experiencias analizadas. Tienen una función clara de soporte a la intervención, y permiten dar calidad y éxito al trabajo. Como ya se ha descrito, pueden dirigirse a los distintos grupos que forman la comunidad educativa, así como adoptar diferentes formas (talleres, formaciones, encuentros, juegos, proyectos, grupos de trabajo...), y darse tanto en la educación formal, como no formal. Siempre teniendo como base la intervención educativa transformadora de la realidad, y el flamenco como aliado en este cambio social.

\section{Bibliografía}

Aix García, Francisco (2014). Flamenco y poder: un estudio desde la sociología del arte. Madrid: Fundación SGAE.

Arnheim, Rudolf (1993). Consideraciones sobre la educación artística. Barcelona. Editorial Paidós.

Barragán, José Ma y Moreno, Ascensión (2004). Experiencia artística y producción cultural, ámbitos para la intervención socioeducativa. Educación Social (28) pp. 19 - 39.

Carnacea, Ángeles y Lozano, Ana (Coords.) (2011): Arte, intervención y acción social. Mediaciones Sociales 9, (II), pp. 187-191.

Cruces Roldán, Cristina (2002). Más allá de la música, Antropología y Flamenco (I). Sevilla. Editorial Signatura de flamenco. 
(C) Servicio de Publicaciones de la Universidad de Murcia

http://revistas.um.es/flamenco

Davila, Mónica (2000). Indicadores de género: guía práctica. Instituto Andaluz de la Mujer. Recuperado de https://cutt.ly/jYo2zu7.

De las Heras, Bárbara (2013). Acercamiento al estudio del baile flamenco desde el ámbito de la educación no formal. Encuentro Plataforma independiente de estudios Flamencos Modernos y Contemporáneos. Sevilla (19 de noviembre). Recuperado de http://ayp.unia.es/dmdocuments/ses0601.pdf.

FAntova, Fernando (2007). Repensando la intervención social. Documentación social (147), pp. 183-198.

Florido, David y Reigada, Alicia (2015). Flamenco y poder. Un estudio desde la sociología del arte [reseña]. Revista Andaluza de Antropología 9, pp. 222-231. Recuperado de https://cutt.ly/MYYguM7.

FreIRE, Paulo (1996). Pedagogía del oprimido. México: Editorial Siglo XXI.

García del Villar Balón, Reyes (2005). Los métodos de la Antropología y la Literatura. Disparidades, Revista de Antropología, 1, pp. 43-58.

Grötsch, Kurt y De las Heras, Bárbara (2010). Los caminos terapéuticos del flamenco. Sevilla: Museo del Baile Flamenco; Madrid: Ecobuk.

Hargreaves, Andy (1998). Profesorado, cultura y postmodernidad. Madrid: Morata.

Hammersley, Martyn y Atkinson, Paul (1994). Métodos de investigación etnográficos. Barcelona: Paidós.

Kisnerman, Natalio (1998). Pensar el Trabajo Social. Buenos Aires-México: Ediciones Edward. Recuperado de https://cutt.ly/zYo3m3e.

KLEIN, Jean Pierre (2006). La creación como proceso de transformación. Papeles de Arteterapia y Educación Artística para la Inclusión Social (1), pp. 11-18.

LAVAUR, Louis (1999). Teoría romántica del cante flamenco: raíces flamencas en la coreografía romántica española. Sevilla: Signatura.

Montenegro, Marisela (2001). Conocimientos, agentes y articulaciones: una mirada situada a la Intervención Social. Athenea Digital (1). Recuperado de: https://cutt.ly/gYYEufG.

Moreno, Isidoro (2012). La identidad cultural de Andalucía. Moreno, Isidoro y Agudo, Juan (Coords.). Expresiones culturales andaluzas. Sevilla: Aconcagua, pp. 11-33.

Moreno GonzÁlez, Ascensión (2010). La mediación artística: un modelo de educación artística para la intervención social a través del arte. Revista Iberoamericana de Educación (OEI), (52/2), pp. 1- 9.

Moreno, Tiburcio. (2003). Replantear el cambio educativo, un enfoque innovador. Perfiles educativos (108), pp. 117-121. Recuperado de https://cutt.ly/8Yo8Qu6.

Murguialday, Clara; VÁzQuez, Norma y GonzÁlez, Lara (2008). Un paso más: evaluación del impacto de género. Barcelona: Cooperacció. Recuperado de https://bit.ly/3pLcFbS.

Pope, Benjamin (1979). The mental health interview: research and application. New York: Pergamon Press.

Rodríguez Ramos, Antonio Manuel (2018). Flamenco: arqueología de lo jondo. Córdoba: Almuzara.

Rubio, María José y VARAs, Jesús (1997). El análisis de la realidad en la intervención social: métodos y técnicas de investigación. Madrid: Editorial CCS.

Steingress, Gerhard. (2006). Sociología del Cante Flamenco. Sevilla: Signatura.

VElAsCo, Honorio y DíAz DE RADA, Ángel (2006). La lógica de la investigación etnográfica: un modelo de trabajo para etnógrafos de la escuela. Madrid: Editorial Trotta.

Washabaugh, William (2005). Flamenco: pasión, política y cultura popular. Barcelona. Paidós. 